\title{
Development and Testing of Hyperbaric Atomic Force Microscopy (AFM) for Biological Applications
}

\author{
Dominic D'Agostino*, Dean Jay* and Helen McNally** \\ *University of South Florida, Molecular Pharmacology and Physiology \\ **Purdue University, Electrical and Computer Engineering Technology
}

Since its development in 1986, atomic force microscopy (AFM) continues to evolve in design and application. Within the last decade the use of AFM for biological applications has grown considerably. To date, almost all biological applications have been performed under ambient conditions. There exists no commercially available research AFMs designed for use at hyperbaric pressures, although a custom-design AFM has been used to study materials at high pressure and temperature [1]. In addition, investigators have made considerable progress in the design and application of ultra high vacuum (UHV) AFM to achieve atomic resolution and lower spectral noise density [2]. An environmental chamber for an AFM was developed and this is capable of measurements at vacuum and controlled humidity [3]. These systems demonstrated the use of AFM outside of ambient conditions, but they were not designed for studying biological samples at hyperbaric pressures. The ideal microscopy system for biological samples at hyperbaric pressure would integrate AFM and fluorescence microscopy and provide stable measurements at physiologically relevant pressures during compression and decompression.

In this study a commercially available AFM (Veeco Bioscope SZ) was installed and tested inside a custom-designed hyperbaric chamber (Figure 1A-C). This report lists the details of developing and testing hyperbaric AFM to study the effects of hyperbaric gases on cellular structure, viscoelasticity and function. The pressure vessel was designed to accommodate a variety of imaging equipment and ensures full functionality at ambient and hyperbaric conditions ( $\leq 6.8$ atmospheres absolute (ATA); 85 PSIG). Electrical, gas and fluid lines were installed to enable remote operation of instrumentation under hyperbaric conditions, and to superfuse viable biological samples with gas-equilibrated superfusate and/or drugs. Results of AFM testing using a calibration grid demonstrate subnanometer resolution at hyperbaric pressure (Figure 2). These observations were confirmed in dry scans and fluid scans, in both contact mode and intermittent contact mode (e.g. tapping mode). Furthermore, AFM scanning was possible during compression and decompression. Installation of a temperature regulation system for cooling and heating the chamber environment was necessary, especially when scanning during a more rapid compression ( $>4 \mathrm{PSI} /$ minute) with a thermally conductive gas (e.g. Helium). AFM system noise ranged between 0.10 and $0.25 \mathrm{~nm}$ at ambient and hyperbaric pressures. There was a trend for less noise under hyperbaric pressures between 15 and 30 PSIG, and this occurred with a variety of hyperbaric gases ( $\mathrm{He}, \mathrm{N}_{2}$, air).

In summary, we describe the development of AFM for use inside a sealed hyperbaric pressure chamber (patent pending). The particular AFM is mated with a fluorescence microscope (Nikon TE2000E), thus expanding the AFM capability for use in biological preparations. The design of the chamber enables easy use of this microscopy system under ambient conditions, and by those unfamiliar with hyperbaric imaging. The microscopy system is 
specifically designed to study living cells under hyperbaric conditions that occur during hyperbaric oxygen therapy, as well as pathological conditions of CNS and pulmonary $\mathrm{O}_{2}$ toxicity, decompression sickness, exposure to increased pressure (e.g., intracranial hypertension) as well as the anesthetic potency of gases (e.g. $\mathrm{N}_{2}$ narcosis).

\section{References}

1. S.R. Higgins et al., Review of Scientific Instruments. 69 (1998) 2994-2998.

2. M.K. Iwatsuki et al., Microsc Microanal. 5;3 (1999) 208-215.

3. J.K. Lievonen et al., Rev Sci Instrum 78(4) (2007) 043703.

4. This research is supported by the Defense University Research Instrumentation Program (DURIP) grant N000140510519 (JBD); ONR grant N000140610105 (DPD), ONR-DURIP equipment grant N000140210643 (JBD)
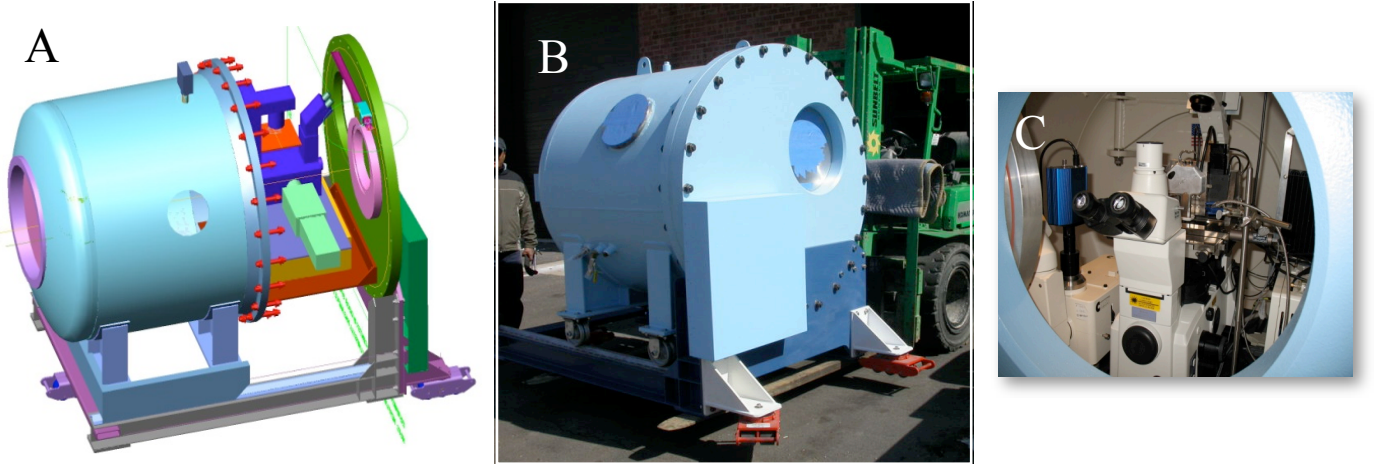

Figure 1. A) CAD drawings of the hyperbaric chamber design before fabrication by Reimers Systems Inc (RSI). B) Hyperbaric chamber after fabrication (photograph from RSI). C) View of Veeco Bioscope SZ through the front sliding chamber door. Open-chamber design allows for installation of equipment and once bolted shut the front and back doors allow for sample placement and easy access to equipment.

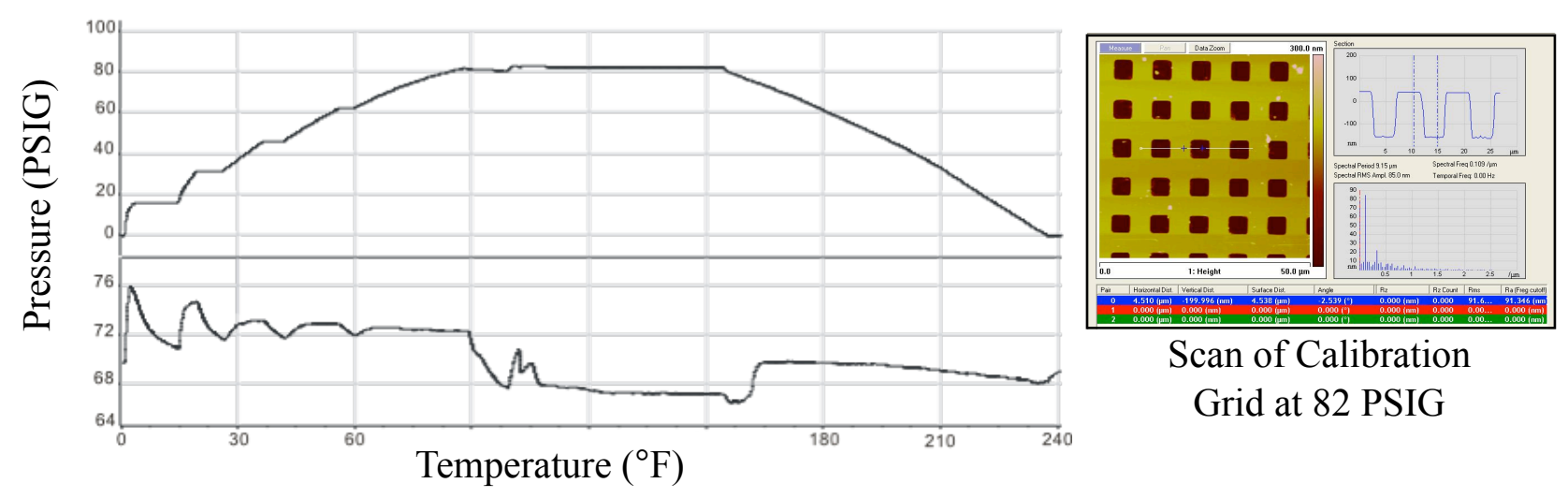

Figure 2. AFM measurements were made at graded levels of pressurization to assess noise and thermal stability under hyperbaric pressure. Changes in temperature were proportional to rate of pressurization and can be minimized with slower compression and decompression. Section analysis of $200 \mathrm{~nm}$ deep pits in a calibration grid was used to demonstrate subnanometer resolution at near maximum working pressure (85 PSIG). 